\title{
Intermediale Elemente in „Das kunstseidene Mädchen“: Verzerrte Selbstwahrnehmung als Folge sozialer Leitbilder der Gesellschaft
}

\author{
Merve Cihangir (iD), İstanbul
}

doi https://doi.org/10.37583/diyalog.1030299

\begin{abstract}
Deutsch)
In der folgenden Studie soll der Roman „Das kunstseidene Mädchen“ (1932) verfasst von Irmgard Keun unter besonderer Berücksichtigung der intermedialen Aspekte untersucht werden. Ziel ist hierbei dem in der Türkei kaum gewürdigten Werk von Irmgard Keun an Sichtbarkeit zu verhelfen als auch in einem intermedialen Rahmen zu betrachten und ferner die negativen Einflüsse der Massenmedien anhand des Fallbeispiels „Doris“ stellvertretend für junge Frauen im 21. Jahrhundert darzustellen. Hierbei soll die Intermedialität und die leserbezogene Methode Richtlinien dieser Arbeit darstellen. Verschiedene Medien wie beispielsweise der Film, die Musik und die Werbung werden im Roman hinreichend aufgegriffen. Besonders um 1920, bedingt durch das neue Frauenbild, aus welchem durch die Medien ein enormer Umschwung der Geschlechterverhältnisse resultierte und außerdem die Gleichberechtigung zwischen Mann und Frau beeinflusste, zeigt sich eine deutliche Wendung der Frau des 20. Jahrhunderts. Medienpersönlichkeiten werden weitgehend zu Idealen der jungen Frauen, was in der Fallstudie Doris eine Schlucht zwischen Realität und Fiktion zur Folge hat. Diese Studie beabsichtigt die negativen Folgen der durch die Massenmedien vermittelten Schönheitsideale aufzuführen und in diesem Rahmen ein gegenwärtiges, soziales sowie zeitloses Problem vor Augen der Leser zu führen.
\end{abstract}

Schlüsselwörter: Keun, Intermedialität, Massenmedien, Schönheitsideale.

\section{Abstract (Englisch)}

Intermedial elements in “The Artificial Silk Girl”: Distorted self-perception as a result of society's social models

In the following study, the novel "The Artificial Silk Girl" (1932) written by Irmgard Keun will be examined with special regard to intermedial aspects. The aim is to give visibility to Irmgard Keun's work, to which no attention was paid in Turkey, as well as to look at it in an intermedial framework and furthermore to present the negative influences of the mass media using the case study "Doris" as a representative of young women in the 21 st century. The intermediality and the reader-related method will be the guidelines of this work. Various media, such as film, music and advertising, are sufficiently addressed in the novel. Especially around 1920, due to the new image of women, which caused an enormous change in gender relations through the media and also influenced the equality between men and women, a clear turn of the woman of the 20th century becomes apparent. Media personalities largely become ideals for young women, resulting in a chasm between reality and fiction in the case study Doris. The aim is to

Einsendedatum: 19.09.2021

Freigabe zur Veröffentlichung: 01.12.2021 
list the negative consequences of the ideals of beauty conveyed by the mass media and, within this framework, to bring a contemporary, social as well as timeless problem before the readers' eyes.

Keywords: Keun, intermediality, mass media, ideals of beauty. 


\section{EXTENDED ABSTRACT}

For centuries, women have been under constant social pressure of the ideal of beauty, with standardization through the mass media usually in the foreground. While women were still overshadowed by their husbands and guardians during the First World War, the 20th century finally saw a decisive change in the system: women transformed themselves from inferior and opinionless companions of men to modern, socially independent personalities and at the same time gained equal rights in marriage. Finally, the term "new woman" established itself, first appearing in the German women's movement of the late 19th and early 20th centuries and embodying the future political, social and personal emancipation of women.

With the association of the new social type of working woman that emerged in the 1920s the values of sociality and emancipation recede into the background and visual or fashionable characteristics of women henceforth become the central issue. In this context, there is clear pressure from the mass media, which form an ideal of beauty that young women often compulsively try to conform to. This problem is still relevant today, especially through social networks such as TikTok, Pinterest or Instagram. The result is a distorted self-image and often a mental illness of young women - the protagonist of the novel "The Artificial Silk Girl" is also under enormous pressure from the mass media and suffers from a clouded self-perception. She creates an illusory world for herself through a cinematic view of life, which makes her the protagonist of her own life but leaves little room for reality. Almost 90 years earlier, Irmgard Keun already formulates a social problem that is still relevant today for young women who are under pressure from a uniform ideal of beauty.

The new image of women is disseminated in numerous media such as magazines, advertisements, films and entertainment literature, with appearance gaining particular importance. Feminine curves are no longer the beauty ideal of the time, instead women have to be slim and boyish, bodies with narrow hips become the new trend. A beauty trend that is all too reminiscent of the current power of social media and points to a long-standing problem of young women.

The following study will both examine these aspects in the novel "The Artificial Silk Girl" and consider their impact on the protagonist and the contemporary problem of young women in the light of the enormous power of mass media. It will be shown that the so-called upward comparison is at the core of the standardization of social models in both the 20th and 21st centuries. There is no question that the power of the media continues to influence women's selfimage, even though there are almost 90 years between the literary example of "The Artificial Silk Girl" and today's society. However, the most important point that still runs like a thread through our society today is the widespread ideal of beauty, especially in printed advertising media and social platforms. Young women can identify with the enormous pressure from the media that the protagonist feels. Often, today's women do not look at themselves like a conventional human being but look through the object of a camera and seem to constantly criticise themselves, which often leads to mental illnesses such as anorexia and bulimia. The interaction of the media leads to a special closeness to reality, as the use of a wide variety of media appeals to the reader's imagination and thus promotes the ability to perceive. Overall, the work "The Artificial Silk Girl" is embellished with the most diverse media. The act of reading is repeatedly accompanied by snapshots, camera perspectives, musical interludes and references to fashionable ideals, with media always interacting with literature. It should be emphasized that the upward comparison in this framework functions as a timeless motif in the novel and in the present, and in this sense the standardization of beauty ideals must be prevented. The aim is to list the negative consequences 
of the ideals of beauty conveyed by the mass media and, within this framework, to bring a contemporary, social as well as timeless problem before the readers' eyes. 


\section{Einleitung}

Frauen stehen bereits seit Jahrhunderten unter dem sozialen Druck des Schönheitsideals, besonders deutlich wird dies mit dem Aufkommen des neuen Frauenbildes aus den USA in den 1920er Jahren. Während durch den ersten Weltkrieg Frauen noch im Schatten ihres Ehemannes, Vormundes standen, kommt es schließlich im 20. Jahrhundert zum entscheidenden Umschwung des Systems, die Frau wandelt sich von der unterlegenen und meinungslosen Gefährtin des Mannes in eine moderne, sozial unabhängige Persönlichkeit und gewinnt gleichzeitig an Gleichberechtigung in der Ehe. Bei der neuen Frau handelt es sich nicht um einen soziologisch beschreibbaren Typus, sondern um ein Leit- und Werbebild der Medien. Der Begriff Neue Frau tritt erstmals in der deutschen Frauenbewegung des späten 19. und frühen 20. Jahrhunderts auf, welche die zukünftig politische, soziale und persönliche Emanzipation der Frau verkörpert.

Der neue Sozialtypus der weiblichen Angestellten wurde in den zwanziger Jahren mit dem amerikanischen Image der modernen berufstätigen Frau, der sogenannten neuen Frau verbunden. Die Werte der Sozialität und Emanzipation rückten in den Hintergrund und visuelle, beziehungsweise modische Eigenschaften der Frau wurden fortan zum Zentralthema. In diesem Rahmen zeigt sich ein deutlicher Druck der Massenmedien, die ein Schönheitsideal formen, dem junge Frauen häufig zwanghaft zu entsprechen versuchen. Die Folge ist ein verzerrtes Selbstbild und häufig eine psychische Erkrankung junger Frauen - auch die Protagonistin des Romans „Das kunstseidene Mädchen“ steht unter diesem enormen Druck der Massenmedien und leidet an einer getrübten Selbstwahrnehmung. Sie formt sich durch einen filmischen Blick auf das Leben eine Scheinwelt, die sie zur Hauptrolle ihres eigenen Lebens macht, jedoch der Realität keinen Raum lässt. Irmgard Keun formuliert bereits knapp 90 Jahre zuvor ein soziales Problem junger Frauen, die unter dem Druck eines einheitlichen Schönheitsideals stehen.

Das neue Bild der Frau erfährt Verbreitung in zahlreichen Medien wie Zeitschriften, Reklame, Filme und Unterhaltungsliteratur, Äußerlichkeiten gewinnen hierbei an großer Bedeutung. Mode dient nicht mehr zur Vorzeige und bricht mit der Korsetttradition, immer mehr Frauen tragen zweiteilige Anzüge und Herrenkostüme. Auch weiblich runde Körper sind nicht mehr das Schönheitsideal der Zeit, sportlich schlanke Körper mit schmalen Hüften werden zum neuen Trend. Es ist ein Schönheitstrend, der nur zu stark an die gegenwärtige Macht der sozialen Medien erinnert und auf ein langlebiges Problem junger Frauen hinweist.

Die folgende Studie soll die aufgeführten Aspekte im Roman „Das kunstseidene Mädchen" sowohl untersuchen als auch ihre Folgen auf die Protagonistin und die gegenwärtige Problematik junger Frauen im Lichte der enormen Macht der Massenmedien betrachten. Hierbei soll sich zeigen, dass der sogenannte Aufwärtsvergleich sowohl im 20. als auch im 21. Jahrhundert den Kern der Normierung sozialer Leitbilder darstellt. Obgleich zwischen dem literarischen Beispiel „Das kunstseidene Mädchen“" und der heutigen Gesellschaft fast 90 Jahre liegen, prägt die Macht der Medien nach wie vor die Selbstwahrnehmung von Frauen 


\section{Intermedialität}

In einem Zeitalter der fortgeschrittenen Technologien und der Begeisterung der Gesellschaft gegenüber Medien, wie Film, Musik, Fernsehen usw. erscheint es fast unmöglich diese Komponenten voneinander und auch von der Literatur $\mathrm{zu}$ isolieren. Medienplattformen und die zunehmende Digitalisierung von Printmedien werden im 21. Jahrhundert zu einem unverzichtbaren Bestandteil unseres Lebens. Oftmals ist uns gar nicht bewusst, dass diese Medien sich auch in der Literatur zu etablieren wissen. Seit Anfang der 90er Jahre stehen wir dem sogenannten Begriff der Intermedialität gegenüber, welcher allgemein ausgedrückt, als Sammelbegriff für verschiedene Phänomene, die mehrere Medien involvieren steht, und somit den Terminus der Medien - Interferenz ablöst (vgl. Zemanek 2012: 166). Ersel Kayaoğlu hingegen erklärt, dass die Techniken, Themen und Erzählstrategien eines jenes Mediums wiederum ein anderes beeinflussen und durch eine Art Nachahmung sowie das Aufgreifen dieser Techniken im fremden Medium eine Verbindung entstehe, die auf die Beeinflussung und das gegenseitige Einwirken der Medien zurückzuführen sei (vgl. 2009: 9). Allgemein ausgedrückt, handle es sich bei der Intermedialität, um die Hybridisierung zwei konventionell voneinander getrennter Medien, in einem Gesamtkunstwerk (ebd.: 9).

Irina Rajewsky spricht ebenfalls von einer Hybridisierung, welches das umfassende Phänomen der Grenzüberschreitung verdeutlicht, wodurch sich mediale Ausdrucksformen, aufeinander zu bewegen, „sich mischen“, ,gegenseitig durchdringen“ und „aufeinander Bezug nehmen“ (2002: 1). Ihren Niederschlag finde die Entwicklung der Intermedialität in ,immer vehementer vorangetriebener Forderung nach einer Interdisziplinarisierung der Wissenschaften und nicht zuletzt in zahlreichen Forschungsund Medienkunstprojekten“ (Rajewsky 2002: 1). Rajewsky unterscheidet hierbei zwischen drei intermedialen Systemreferenzen: die explizite Systemerwähnung, welche lediglich die schlichte Erwähnung eines Mediums verdeutlicht und die Systemerwähnung qua Transposition, bestehend aus Elementen der Evokation, Imitation und (Teil-) Reproduktion, welche einen zunehmend illusionsbildenden Charakter, insbesondere den sogenannten ,Als- ob“ Charakter innehat (ebd.: 79 ff.). Letzterer ist an das filmbezogene Schreiben geknüpft und trägt durch Evokation oder Simulation zur Illusionsbildung im Leseakt bei. Da Autoren nicht über die Techniken des Films wie beispielsweise Zoom oder Zeitlupe verfügen, erzeugen sie demnach durch filmbezogenes Schreiben einen „Als

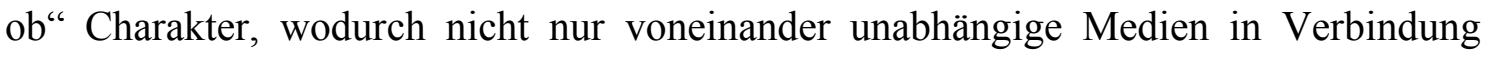
gesetzt werden, sondern auch zur Illusionsbildung beitragen wird.

Eine besonders grenzüberschreitende Rolle im Rahmen der Intermedialität nimmt das Element der Musik ein. Während Rajewsky dieses der Transmedialität zuordnet (Interaktion von medienunspezifischen Phänomenen), spricht Nicola Gess hierbei von einem fremdmedialen Bezug, den sie unter dem Begriff Medientransformation zusammenfasst (vgl. Lubkoll 2017: 79). In Anlehnung an Werner Wolf und Rajewksy, schlägt Gess hierbei die Begriffe „telling“ und „showing“ vor: Telling (explizite Referenz) kann hierbei als das Erzählen über Musik gedeutet werden, ebenso ist hierbei die Erwähnung von zeitgenössischen Komponisten, Musikern und ihre Bedeutung für die Musik relevant sowie erwähnenswert. Bekannte Beispiele dafür finden sich bereits in der 
Epoche der Romantik (Novalis, E.T.A. Hoffmann, Tieck) (ebd.: 82 ff.). Showing (implizite Referenz) bzw. verbal music bezieht sich hingegen auf die Narration von existierenden oder fiktiven Musikwerken in der Literatur.

Grob ausgedrückt beschreibt die Intermedialität das Zusammenspiel verschiedener Medien, unterstützt durch ihre technischen Entwicklungen und die Wechselbeziehungen zwischen Schrift und Bild.

\section{Intermediale Aspekte in „Das kunstseidene Mädchen“}

„Das kunstseidene Mädchen“ (1932) von Irmgard Keun erzählt von den Lebensumständen der achtzehnjährigen Doris und ihrem Wunsch ein Glanz ${ }^{1}$ zu werden. Nach unsittlichen Annäherungsversuchen ihres Chefs wird sie gekündigt und beginnt eine Ausbildung zur Schauspielerin im Theater. Nach Lügen, Intrigen und letztendlich einem Diebstahl flieht sie nach Berlin, um dort ihrem Wunsch ein Glanz zu werden nachzugehen. Dort kommt sie vorübergehend bei einer Bekannten unter und lässt sich von Männerbekanntschaften finanzieren. Ihr stetiger Begleiter ist ein $\mathrm{Feh}^{2}$, der eine Brücke zum luxuriösen Leben darstellt, welches sie sich als angehender Glanz erträumt. In Berlin wird Doris mit den Problemen der Anonymität, Wohnungslosigkeit und Arbeitslosigkeit konfrontiert, weshalb sie an einer selbsterzeugten Schein- bzw. Filmwelt festhält und Halt im Leben sucht. Der Roman endet ohne eine Aussicht auf Hoffnung. Obgleich Keun nur wenig Privates über sich preisgegeben hat, sind die autobiographischen Züge in ihren Werken nicht zu übersehen. Die Autorin selbst behauptete, dass sie nach Abschluss der Privatschule eine Hauswirtschaftsschule besucht habe. Auch datierte sie ihr Geburtsjahr auf 1910 um und behauptete mit 15 Jahren eine Schauspielausbildung begonnen zu haben (Marchlewitz 1999: 22). Keun habe sich das Leben als Schauspielerin nicht als harte und disziplinierte Knochenarbeit, sondern als freie und unabhängige Existenz vorgestellt, wie sie es wenig später durch die Protagonistin Olga aus „Gilgi, eine von uns“ (1931) beschrieben hat.

\section{Elemente des Kinos}

Und ich denke, dass es gut ist, wenn ich alles beschreibe, weil ich ein ungewöhnlicher Mensch bin [...] ich will schreiben wie Film, denn so ist mein Leben und wird noch mehr so sein. [...] Und wenn ich später lese ist alles wie Kino - ich sehe mich in Bildern. (Keun 2008 [1932]: 8)

Mit diesen Worten beginnt das Werk und zeigt schon zu Anfang unübersehbare Elemente der Intermedialität. Doris, die Protagonistin des Werkes verzeichnet diese Worte in einem Tagebuch, welches äußerlich durch die Farbsymbolik der weißen Tauben auf schwarzem Untergrund an das filmische ,schwarz-weiß“ zu erinnern scheint. In diesem Sinne ließe

\footnotetext{
${ }^{1}$ Die Eigenschaften eines Glanzes stehen im Roman analog zu einer achtungswürdigen Persönlichkeit. Doris, die Protagonistin sieht dies als anzustrebendes Ideal: „Ich will so ein Glanz werden, der oben ist. Mit weißem Auto und Badewasser, das nach Parfüm riecht, und alles wie Paris. Und die Leute achten mich hoch, weil ich ein Glanz bin" (Keun 2008 [1932]: 26).

${ }^{2}$ Feh wird als Synonym für Pelzmantel genutzt.
} 
sich also nicht ausschließlich von einem Tagebuch, sondern auch von einer Art Drehbuch sprechen, da ihr Leben Züge eines Films trägt. Hierbei wird die These Rajewskys bestätigt, welche besagt, dass verschiedenste Medien sich vermischen bzw. aufeinander Bezug nehmen (vgl. 2002: 1). Die Grenzüberschreitung von Literatur und Kino zeigt sich daher in einer Art hybriden Verschmelzung der genannten Komponenten. Auch die Tagebucheintragungen des jungen Mädchens sind gekennzeichnet durch Momentaufnahmen, gedankliche Dialoge und Beschreibungen detailgetreuer Bilder, die an das Medium des Films erinnern. Erneut zeigt sich, dass Erzählstrategien des Films die Literatur zu beeinflussen scheinen, aus der Nachahmung sowie dem Aufgreifen verschiedenster Medien untereinander entsteht, wie durch Kayaoğlus These bestätigt wird, eine Verbindung zwischen verschiedenen Medien untereinander, die auf ihre gegenseitige Einwirkung zurückzuführen ist (vgl. 2009: 9). So agiert Doris weniger als herkömmliche literarische Figur, sondern schafft durch ihre Beschreibungen aus ihrem Dasein eine Art Filmcharakter: „Der Blick auf sich selbst ist an filmischen Wahrnehmungsweisen geschult" (Barndt zit. n. Fast 2015: 25). Sie wird durch ihren Kamerablick gleichzeitig zur Regisseurin und Schauspielerin ihres eigenen Lebens. Beispielsweise lässt sie bei einer Taxifahrt ihren Blick kameraartig schweifen und beschreibt in aneinandergereihten Bildern ihre Umgebung, dies erinnert nur zu deutlich an eine Art Kamerafahrt:

\begin{abstract}
Und bin heute allein Taxi gefahren wie reiche Leute - so zurückgelehnt und den Blick meines Auges zum Fenster raus - immer an Ecken Zigarrengeschäfte - und Kinos - und der Kongreß tanzt - Lilian Harvey, die ist blond - Brotläden und Nummern von Häusern mit Licht und ohne - und Schienen - [..] blaue Lichter, rote rote Lichter, viele Millionen Lichter - Schaufenster - Kleider - aber keine Modelle - andere Autos fahren manchmal schneller - [...]. (Keun 2008 [1932]: 127)
\end{abstract}

Im obigen Zitat zeigt sich die Verschmelzung der Komponenten Film und Literatur, da Aneinanderreihungen ihrer Momentaufnahmen durch kurze Ortsangaben, an wechselhafte Wahrnehmungen einer Taxifahrt erinnern, die sich aufgrund der Geschwindigkeit im Sekundentakt wechseln. Da hierbei in Anlehnung an Rajewsky die Autorin des Romans nicht über die Instrumente des Films verfügt, zeigt sich durch das filmbezogene Schreiben die Evokation bzw. Simulation der Kameratechniken, welche den sogenannten „Als ob" Charakter im Roman erzeugen und folglich zur Illusionsbildung im Leseakt beitragen (vgl. 2002: 85). In der Tat trifft der Leser an dieser Stelle auf ein Dilemma des filmbezogenen Schreibens, die Wahrnehmung im Leseakt ist subjektiv geprägt an die Vermittlung des Autors geknüpft, demnach ist nach Schmidt nur von einer bedingten Wirklichkeit die Rede ,,in Wirklichkeit handelt es sich doch nur um eine Illusionsvermittlung des Autors mit dem er den Ablauf eines filmischen Ablaufs suggeriert" (Schmidt zit. n. Rajewsky 2002: 85).

Äußerlich beschreibt die Protagonistin sich durch einen Vergleich zur Filmschauspielerin Colleen Moore: „Ich sehe aus wie Colleen Moore“ (Keun 2008 [1932]: 4), wodurch eine berühmte Filmpersönlichkeit mit einer literarischen Fiktion zu verschmelzen scheint. An dieser Stelle zeigt sich deutlich, dass die Realität und Fiktion im Werk hybridisiert werden und im Roman als Gesamtwerk erscheinen. Auch bedient sie sich eines Vergleiches zur Schauspielerin Marlene Dietrich: „Und ich schaue wie 
Marlene Dietrich so mit Klappaugenmarke“ (ebd.: 13). Zum einen zeigt sich hierbei der Präsenz realer Persönlichkeiten im narrativen Bezug, weshalb an dieser Stelle von einer Einzelreferenz als auch eine Transformation einer realen Persönlichkeit auf die Fiktion die Rede sein kann. Durch diese Beschreibungen wird nur unschwer deutlich, dass ihr Wunsch ein Glanz beziehungsweise ein anzustrebendes Ideal der Gesellschaft zu werden, einen starken Einfluss auf das Selbstgefühl der Protagonistin hat: „Ich will so ein Glanz werden, der oben ist. Mit weißem Auto und Badewasser, das nach Parfüm riecht, und alles wie Paris. Und alle Leute achten mich hoch, weil ich ein Glanz bin [...]" (ebd.: 26). Ihr Wunsch einen Idealtypus zu erfüllen, ist eng mit der Popularität der Massenmedien verknüpft, die Protagonistin erschafft ein Leben im Film mit ihrer eigenen Fantasie. Auffallend ist, dass sie sich wie durch das Objekt einer Kamera nur oberflächlich zu betrachten scheint, dies spiegelt sich deutlich in ihrer Körperwahrnehmung wider. Durch die medialen Techniken ist sie im Stande eine andere Person zu werden. Sie versucht somit dem weitverbreiteten Bild der Neuen Frau zwanghaft zu entsprechen. Dazu gehört auch ein abgemagerter Körper-Schönheit zeigt sich in diesem Sinne lediglich definiert durch die Bestimmungen der Medien. Ihr Körper ließe sich demnach als Medium beschreiben, welches durch einen Kamerablick betrachtet und stets mit der medialen Vorgabe verglichen wird. Besonders in ihren Beschreibungen, die sie dem blinden Brenner ${ }^{3}$ bietet, zeigt sich eine deutliche filmische Wahrnehmung, sie: ,packt ihre Augen vor ihm aus" (ebd.: 107) und zeigt ihm durch eine Art Kino das Leben in der Großstadt Berlin. Die Stadt wird durch die Erlebnisse in bunten Bildern dargestellt, wobei bruchstückhaft und in nicht zusammenhängenden Sätzen gesprochen wird: „Da war ich ein Film und eine Wochenschau“" (ebd.: 126).

Die Macht der Massenmedien ist auch im 21. Jahrhundert weiterhin die Richtline junger Frauen, soziale Plattformen verkörpern ähnlich wie das neue Bild der Frau, ein Bild der Perfektion, welches besonders bei jungen Frauen derart ausartet, dass die Körperwahrnehmung verzerrt wahrgenommen wird. Krankheiten wie Anorexie und Bulimie können als Beispiel für psychische Erkrankungen und verzerrter Körperwahrnehmung genannt werden ${ }^{4}$.

\section{Kinobesuche}

Doris besucht im Roman gerne das Kino, hierbei kommt besonders die Unzufriedenheit mit ihrem eigenen Leben zum Vorschein. Sie muss die Diskrepanz zwischen der medialen Scheinwelt und dem wirklichen Leben bitter zu spüren bekommen. Sie vergleicht die eigene mit der Filmwelt und muss erkennen, dass diese durch Unwirklichkeit geprägt ist. Auch entspricht sie dem Bild der Neuen Frau nur mit äußerster Mühe. Sie beginnt

\footnotetext{
${ }^{3}$ Handelnde Figur im Roman „Das kunstseidene Mädchen“. Die Romanfigur hat, wie der Name „blinder Brenner" bereits vermuten lässt, eine Sehbehinderung.

${ }^{4}$ Anorexie: Übermäßige Gewichtsabnahme infolge von Unter- und Fehlernährung, organischen od. psychischen Störungen [Magersucht] (Wahrig 2001: 841).

Bulimie: Psychosomatische Erkrankung, bei der versucht wird, Heißhungerattacken mit unkontrollierter Nahrungsaufnahme durch künstlich herbeigeführtes Erbrechen zu korrigieren. [grch. Bulimia „Heißhunger; Ochsenhunger"] (Wahrig 2001: 306).
} 
beispielsweise nach einem Kinobesuch ihr eigenes Leben und ihre Beziehungen zu hinterfragen: „Bin ich ein Kino oder eine Liebe“ (Keun 2008 [1932]: 114) ihr ist somit bewusst, dass das Medium des Films eine Unwirklichkeit beinhaltet, die nicht zur Realität passt. Auch wird durch den Kinobesuch des Films „Mädchen in Uniform“ (1931) ein damals noch als Tabu geltendes Thema, nämlich das der Homosexualität, aufgegriffen: „Wir haben zusammen im Kino gesessen, es war ein Film von Mädchen in Uniform“ (ebd.: 114). Die Ortsbeschreibungen im Roman sind oft an realen Beispielen orientiert, welche dem Medium des Films entsprechen. Beispielsweise beschreibt Doris den Gloriapalast, ein großes Kino, welches die Uraufführungen der Spielfilme in den 1930ern gezeigt hat: „Der Gloriapalast schillert - ein Schloss ein Schloss, es ist aber ein Kino und Kaffee in Berlin W - um die Kirche sind schwarze eiserne Ketten - und drüben das Romanische Kaffee“ (ebd.: 61).

\section{Popularität des Films}

Es handelt sich bei der Verwendung des Mediums Film nicht ausschließlich um eine Filmbegeisterung der Autorin, sondern vielmehr um die weitverbreitete Popularität der Massenmedien um 1920. Das Kino galt damals als ein geselliges Freizeitvergnügen, welches ähnlich wie Fußball und Kneipenbesuche zum Leben der bildungsbürgerlichen Mittelklasse gehörte. Die berühmten Persönlichkeiten werden zu Richtlinien und Identifikationsobjekten der Menschen:

Als Geschöpfe zwischen Illusion und Alltagsrealität trugen die Stars zudem den Film ins tägliche Leben ihrer Zuschauer - mit direkten Folgen für Mode, Auftreten und Lebenswandel. Nicht nur wurden Aussehen und Verhalten der Stars von ihren Fans nachgeahmt, es entstanden sogar universelle Idealtypen durch filmische Vorbilder. (Kaes 1993: 92)

Somit ist die Filmbegeisterung im Werk auf die damalige Popularität der Massenmedien zurückzuführen. Doris kann demnach als eine von vielen verstanden werden, deren Leben durch diese aufkommende Begeisterung beeinflusst wurde. „Ich werde ein Glanz, und was ich dann mache, ist richtig - nie mehr brauch ich mich in Acht nehmen .. nichts kann mir mehr passieren an Verlust und Verachtung, denn ich bin ein Glanz" (Keun 2008 [1932]: 27), ein Glanz zu sein macht eine Frau in den Augen von Doris praktisch unverwundbar, es bedeutet Berühmtheit und Anerkennung von allen Seiten. Auffallend ist jedoch, dass Doris den Glanz als einzigen Weg sieht, Anerkennung von anderen Menschen zu erlangen. Eine weitere Möglichkeit sieht sie in diesem Sinne nicht. Berühmtheit macht einen Menschen in ihren Augen unverwundbar. In der Großstadt Berlin muss sie einsehen, dass die zunehmende Anonymität und Trostlosigkeit dazu führen, dass menschliche Werte wie Zuneigung und Wärme verloren gehen, auch in diesem Fall macht ein Glanz nicht glücklich: „...und es ist kein Glanz und keine Menschen - lauter gestorbene Grabsteine gehen“ (ebd.: 65). Gerade in diesem 
Zusammenhang zeigt sich ihre kindliche Naivität. Sie muss jedoch durch die Begegnung mit Ernst ${ }^{5}$ erkennen, dass ein Glanz nicht maßstabgebend für menschliche Achtung ist.

Wenden wir die Feststellung der Protagonistin auf die heutige Zeit an, so ließe sich behaupten, dass das Werk durch die Einbindung intermedialer Aspekte auch eine Botschaft an das 21. Jahrhundert weitergibt, da der enorme Druck der Massenmedien und seine fatalen Folgen auf junge Frauen oftmals unterschätzt wird. Die Einsicht der Protagonistin „Auf den Glanz kommt es nämlich vielleicht gar nicht so furchtbar an“ (ebd.: 116), erklärt sich als eine Widerstandserklärung gegen die Macht der Schönheitsideale, welche durch Medien vermittelt werden.

\section{Elemente der Musik}

Dem Medium des Films entsprechend, wird die Musik im Hintergrund unterstützend aufgegriffen, um beispielsweise die Gefühle der Protagonistin für den Leser deutlich zu machen. Auch wird der Bezug zu berühmten Komponisten hergestellt, beispielsweise wird der österreichische Komponist Peter Tschaikowski (1840-1893) erwähnt: „Meine Frau liebte Tschaikowski“ (Keun 2008 [1932]: 100). Peter Tschaikowski ist besonders für sein Stück „Swan Lake“ (1876) bekannt, welches sowohl als Theatermusik, als auch als Filmmusik genutzt wurde. Auch der Komponist Franz Peter Schubert (1797- 1828) bleibt nicht unerwähnt: „Schubert, sagte er. Wieso? Gesungen hat sie, wie Schubert komponiert hat" (ebd.: 116). Die Protagonistin bedient sich hierbei eines Vergleichs, wodurch die audiovisuelle Technik aufgegriffen wird. Diese Erwähnungen weisen auf das „telling“ in Anlehnung an Nicola Gess Unterteilung der Musikalisierung in der Literatur hin, hierbei werden oftmals Erwähnungen bezüglich berühmter Musiker, Sänger usw. narrativ im Roman aufgenommen, diese Art der Medientransformation war bereits eine beliebte Methode der Autoren der Romantik (vgl. Lubkoll 2017: 82).

Die Schlagermusik ließe sich als stetiger Begleiter von Doris beschreiben, da dieser mit ihrem eigenen Leben identifiziert wird und ihre Gefühle darstellt. Sie identifiziert sich beispielsweise mit einem Charakter aus dem deutschen Schlager: „Da habe ich ihnen schließlich den Schlager gesungen, von Elisabeth ihre schönen Beine und habe dazu getanzt so von einer Seite zur andern“(Keun 2008 [1932]: 25). Durch diese Beschreibung zeigt sich zum einen die Verschmelzung der jungen Frau mit dem Medium der Musik und zum anderen ihre oberflächliche Körperwahrnehmung, welche sich an Schönheitsidealen der Massenmedien zu orientieren scheint. Weiterhin greift Doris das Lied Wien, du Stadt meiner Träume (1914) von Rudolf Sieczyński auf, als sie alkoholisiert ist:

Wien, Wien nur du allein, Wien - da saßen wir bei dieser Musik aus dem Radio. Ach so schön. Das gibt's nur einmal, das kommt nicht wieder - das ist zu schön um - Wien, Wien nur du allein - Wien, Wien Musik mit dir - in diesem Moment fühle ich mich wie

\footnotetext{
${ }^{5}$ Handelnde Figur in „Das kunstseidene Mädchen“. Ernst lebt getrennt von seiner Ehefrau einige Zeit mit Doris zusammen, durch ihn zeigt die Protagonistin Einsicht und beginnt ihren Traum ein Glanz zu sein, zu hinterfragen.
} 
ein Dichter $[\ldots]$ ich habe einen Schwips, er hat mir Kognak eingeschenkt, ich vertrag keinen Kognak. (ebd.: 115)

Diese Bezugnahme auf das Stück Sieczyńskis ist im Rahmen der intermedialen Referenzen auf die verbal music bzw. die implizite Referenz (showing) nach Ness und die Teilreproduktion (zitieren eines Liedtextes im Roman) nach Rajewsky zurückzuführen (vgl. Wolf 2017: 98). Das Medium der Musik wird im literarischen Werk, ähnlich wie im Film genutzt, um den emotionalen Zustand der Figuren zu vermitteln. Auch an dieser Stelle zeigt sich, dass die Medien Musik und Literatur derart miteinander verschmelzen und somit eine Art Lebendigkeit der literarischen Produktion erzeugen. Lubkoll verweist hierbei auf „Ein höchst interessantes Phänomen bei Darstellungen von Musikwerken in der Literatur ist eine Art doppelte Intermedialität, denn häufig wird beschrieben, wie beim Musikhören Bilder im Inneren der Seele aufsteigen“ (2017 :86). In der Tat zeigt sich auch am Fallbeispiel Doris, dass Musik nicht nur als Flucht aus der Realität gedeutet werden kann, sondern aus intermedialer Sicht eine unterstützende Instanz zum Verständnis der Gefühlslage der handelnden Figuren darstellen kann.

\section{Soziale Leitbilder und verzerrte Selbstwahrnehmung im 21. Jahrhundert}

Die Macht der Medien, insbesondere der sozialen Netzwerke, ist im Zeitalter der Digitalisierung nicht zu unterschätzen.

Während Menschen über das Internet in ständiger Kommunikation stehen, porträtieren soziale Plattformen Prominente, die im Alltag nicht zu erreichen sind. Sogenannte Challenges ${ }^{6}$ gehen viral ${ }^{7}$ auf sozialen Plattformen wie TikTok, Instagram, Pinterest u.a., hierbei werden Schönheitsideale wie beispielsweise thigh gap- ${ }^{8}$ oder A4 skinny waist ${ }^{9}$ - Challenge idealisiert. Diese und ähnliche Internettrends sind häufig mit dem Vergleichsreflex des Menschen verbunden:

Beim Betrachten einer idealisierten Körperdarstellung in den Medien kommt es zum Aufwärtsvergleich (die andere Person wird als attraktiver wahrgenommen), der abhängig vom Individuum zu Frust oder Deprimiertheit über das eigene Aussehen/Körperbild führen kann oder aber dazu, dem Vorbild nachzueifern. Da diese präsentierten Schönheitsideale aber oft für den/ die Normalbürger*in nicht oder nur schwer erreichbar sind (allein schon wegen eingesetzter Bildbearbeitungstechniken), kann Letzteres im schlimmsten Fall zu krankhaftem Verhalten führen (Brosius / Peter 2020: 56).

Dem obigen Zitat ist zu entnehmen, dass der sogenannte Aufwärtsvergleich die idealisierte Köperdarstellung der Medien zur Norm erhebt, der Unzufriedenheit mit dem eigenen Körper folgt demnach zunächst die Frustration und anschließende psychisch

\footnotetext{
${ }^{6}$ Challenge ist im Netz grundsätzlich mit einer Erklärung einer Sozialen Netzwerk - Herausforderung verbunden, Beispiele dafür sind thigh gap - Challenge und die A4 skinny waist - Challenge.

${ }^{7}$ Der Ausdruck ,viral gehen“ geht ursprünglich auf die vakante Verbreitung eines Virus zurück, im Netz beschreibt es die Verbreitung eines Sozialen Netzwerk - Trends.

${ }^{8}$ Die thigh gap - Challenge ist eine Herausforderung in sozialen Netzwerken, Ziel ist es eine Lücke zwischen der Innenseite der Oberschenkel zu erzielen.

${ }^{9}$ Die A4 skinny waist - Challenge ist eine Herausforderung mit dem Ziel eine Taille zu erlangen, die nicht breiter als ein DINA 4 Blatt ist.
} 
veranlagte Verhaltensänderungen, aus welchem eine verzerrte Körperwahrnehmung und im schlimmsten Fall eine Essstörung resultieren kann. Der Aufwärtsvergleich birgt die scheinbare Erkenntnis der Unterlegenheit des Rezipienten, welcher sich im Vergleich zum Ideal als unattraktiv einschätzt. Christian Schemer betont, dass die Reaktion des Rezipienten hierbei stark von dessen Selbstwertgefühl abhängig sei, Personen mit geringem Selbstwertgefühl seien daher unzufriedener und eher von sozialen Vergleichen mit attraktiven Medienakteuren betroffen als Rezipienten mit höherem Selbstwertgefühl (vgl. 2007: 60).

Die besagten Trends, sowie der Einfluss von Massenmedien verdeutlicht, dass sich die literarische Produktion Irmgard Keuns ebenso auf die heutige Zeit projizieren lässt. Obgleich soziale Netzwerke zu Anfang des 20. Jahrhunderts noch nicht verbreitet waren, zeigt sich, dass Frauen sich knapp 90 Jahre nach dem Fallbeispiel Doris weiterhin durch soziale Leitlinien beeinflussen lassen. Der Blick auf den Körper erfolgt sowohl bei Doris als auch in der heutigen Zeit häufig nicht aus einer realistischen Perspektive, sondern meist aus dem Objektiv einer Kamera, welches an der Normierung der Medien orientiert ist. Ziel ist es hierbei in beiden Fällen die Anerkennung der Gesellschaft, um jeden Preis zu erhalten, als Voraussetzung gilt einem Bild $\mathrm{zu}$ entsprechen, welches oftmals nur schwer zu realisieren ist. Makellosigkeit und Unverwundbarkeit gehen in diesem Rahmen Hand in Hand. Die Augen werden vor dem Unschönen, Unattraktiven geschlossen, was zählt ist nur noch die erzeugte mediale Scheinwelt und das Schönheitsideal, dem es um jeden Preis zu entsprechen gilt.

\section{Schlussbemerkung}

Zweifellos nahmen die populären Medien in den zwanziger Jahren einen besonderen Stellenwert ein, demnach zeigt sich die Dominanz des Films, der Musik und der Zeitschriften, besonders deutlich. Auch gegenwärtig beeinflusst die Macht der Medien, insbesondere die der Sozialen Netzwerke häufig das Leben junger Frauen. Filmstars, Models und Internetberühmtheiten gelten auch heute häufig noch als Richtlinien der eigenen Körperwahrnehmung. Demnach bildet sich aufgrund der deutlichen medialen Elemente im Alltag eine Art Schlucht zwischen Realität und medialer Scheinwelt. Im Falle von Doris zeigt sich, dass sie mit äußerster Mühe versucht dem vermittelten Bild der Neuen Frau zu entsprechen. Anerkennung und Bewunderung der Gesellschaft sind hierbei an das Körperbild der jungen Frau geknüpft, weshalb sie ihr Leben nach diesen Idealen richtet. Ihre Fantasie ermöglicht ihr zwar ein Leben wie im Film, jedoch muss sie zu Ende des Werkes einsehen, dass die Abhängigkeit von der Macht des Films bedeutungsloser ist, als sie es zu Anfang glaubte. Somit ist die Flucht in die Welt der Medien im Falle von Doris als Flucht vor der Realität zu deuten. Auch in der Gegenwart stehen weiterhin die sozialen Leitlinien im Vordergrund und werden zudem auch durch

Soziale Netzwerke bekräftigt. Äußerlichkeiten sind weiterhin häufig mit gesellschaftlichen Trends und Vorgaben verbunden, sodass oftmals junge Frauen ähnlich wie im Fall von Doris die Anerkennung der Gesellschaft in Verbindung eines Schönheitsideals sehen. Somit kommen wir zu dem Schluss, dass die Popularität der 
Medien durch Ihren Akt der Verschmelzung mit der Literatur, bei dem vorliegenden Beispiel sehr deutlich erfasst wird und sich auch in der aktuellen Situation junger Frauen widerspiegeln kann.

Die Verwebung der Elemente Literatur, Musik, Film etc. unterstützt nicht nur die Fantasie der Protagonistin, sondern bietet dem Leser eine bunte Welt, gefüllt mit dem Zusammenspiel verschiedenster Medien. Die Gefühle der Protagonistin werden ähnlich wie im Film, durch die entsprechende Hintergrundmusik unterstützt und können in diesem Sinne ebenfalls die Gefühlswelt des Lesers berühren. Der Leser fühlt sich wie im Kino, wenn er mit Doris auf die Leinwand schaut und die Schlucht zwischen Realität und Fiktion hinterfragt. Die mediale Scheinwelt im Roman erinnert jedoch ebenso an die gegenwärtige Macht der sozialen Medien, welche durch ihre Popularität und vorgegebenen Leitbilder häufig ähnliche negative Folgen auf junge Frauen haben kann.

Der wichtigste Punkt jedoch, welcher sich auch heute weiterhin wie ein roter Faden durch unsere Gesellschaft zieht, ist das weit verbreitete Schönheitsideal, besonders in gedruckten Werbemedien und sozialen Plattformen. So kann sich jene junge Frau mit dem enormen Druck der Medien, welchen die Protagonistin empfindet, identifizieren. Oftmals betrachten sich auch Frauen der heutigen Zeit nicht wie ein herkömmlicher Mensch, sondern sie schauen durch das Objekt einer Kamera und scheinen sich stets zu kritisieren, dies hat oftmals psychische Krankheiten wie Anorexie und Bulimie zur Folge. Aus dem Zusammenspiel der Medien ergibt sich besonders die Nähe zur Wirklichkeit, da die Verwendung verschiedenster Medien, die Fantasie des Lesers anspricht und somit die Wahrnehmungsfähigkeit fördert. Insgesamt ist das Werk ausgeschmückt mit verschiedensten Arten von Medien und bewahrt noch heute durch die Normierung sozialer Leitbilder seine Aktualität. Der Leseakt ist stets begleitet durch Momentaufnahmen, Kameraperspektiven, Musikeinlagen und den Verweisen auf Modeideale sowie berühmte Persönlichkeiten, wodurch Medien stets im Zusammenspiel mit der Literatur stehen. Abschließend ist zu betonen, dass der Aufwärtsvergleich in diesem Rahmen als zeitloses Motiv sowohl im Roman „Das kunstseidene Mädchen“ als auch der heutigen Zeit, besonders durch den Einfluss sozialer Plattformen, verstanden werden kann und in diesem Sinne die Normierung von Schönheitsidealen vorzubeugen gilt.

\section{Literaturverzeichnis}

Fast, Evelyn (2015): Das Frauenbild in der Literatur der 1920er Jahre: Die ,Neue Frau ' bei Irmgard Keun, Marieluise Fleißer und Mela Hartwig. Hamburg: Diplomica Verlag.

Kaes, Anton (1993): Film in der Weimarer Republik. In: Jacobsen, Wolfgang u.a. (Hg.): Geschichte des deutschen Films. Stuttgart und Weimar: J.B. Metzler, 38-98.

Kayaoğlu, Ersel (2009): Edebiyat biliminde yeni bir yaklaşım: Medyalararasılık. İstanbul: Selenge Yayınları.

Keun, Irmgard (2008 [1932]): Das kunstseidene Mädchen. Leipzig: Ernst Klett Verlag.

Lubkoll, Christine (2017): Musik in der Literatur: Telling. In: Gess, Nicola / Honold, Alexander (Hg.): Handbuch Literatur \& Musik. Berlin: Walter de Gruyter Verlag, 78 -95. 
Marchlewitz, Ingrid (1999): Irmgard Keun: Leben und Werk. Würzburg: Verlag Könighausen \& Neumann.

Peter, Christina / Brosius, Hans Bernd (2020): Die Rolle der Medien bei Entstehung, Verlauf und Bewältigung von Essstörungen. In: Bundesgesundheitsblatt - Gesundheitsforschung - Gesundheitsschutz $64,55-61$.

Rajewsky, Irina O. (2002): Intermedialität. Tübigen/ Basel: A. Francke Verlag.

Schemer, Christian (2007): Wem Medienschönheiten schaden. Die differenzielle Anfälligkeit für negative Wirkungen attraktiver Werbemodels auf das Körperbild junger Frauen. In: Zeitschrift für Medienpsychologie. 19(2), 58-67.

Wahrig, Gerhard (2001): Wahrig: Deutsches Wörterbuch. München: Bertelsmann Lexikon Verlag.

Wolf, Werner (2017): Musik in der Literatur: Showing. In: Gess, Nicola / Honold, Alexander (Hg.): Handbuch Literatur \& Musik. Berlin: Walter de Gruyter Verlag, 95 -114.

Zemanek, Evi (2012): Intermedialität - Interart Studies. In: Zemanek, Evi / Nebrig, Alexander (Hg.): Komparatistik. Berlin: Akademie Verlag, 159-166. 